Physics International 2 (2): 40-49, 2011

ISSN 1948-9803

(C) 2011 Science Publications

\title{
The Concept of Event-Driven Time
}

\author{
Sergey K. Aityan \\ Lincoln University, \\ 401 15th Street, Oakland, California 94612, USA
}

\begin{abstract}
The most fascinating feature of time is that there is no time at all. This study introduces the concept of event-driven time. Time is considered a count of changes or events that set up a local "clock" in each system. With such an approach, every system runs its own local time. Time does not exist without changes in the system. As two systems interact, they generate a sequence of interaction events that are being added to the internal pool of events in each system. Any observation is made with the local time of the observer system and this fact changes the observer's local time by adding observation events to the observer's time count. Local time in any finite and closed system is finite and obeys the saturation principle due to limitations of the event counting capacity of the system. Traditional continuous time is a convenient approximation for the enormous number of events occurring in our world that set up our local "clock". The event-based approach does not conflict with modern physics but proposes a new view of the fundamental notion of time and brings us one step closer to understanding the world in which we live. The greatest mystery of the notion of time is that there is no time at all.
\end{abstract}

Key words: Time, nature of time, event-time, quantum time

\section{INTRODUCTION}

Time is the most fascinating feature of matter. The concept of time has always been a cornerstone of all theories in physics and other disciplines of natural sciences. In the past, people thought of time as a universal outside clock ticking independently of matter and space, just tracking all processes in the universe. Isaac Newton in his Principia, Newton, 1687 (Newton and Reviewer, 1999) wrote,- "absolute, true and mathematical time, of itself and from its own nature, flows equably without relation to anything external."

Later, as the theory of relativity (Einstein, 2004) emerged, the concept of time has become more closely tied to matter and space. One of two fundamental postulates of relativity is based on the Lorentz transformation and declares the invariance of the speed of light in a vacuum. It states that in vacuum, light propagates with respect to any inertial frame and in all directions with the universal speed $\mathrm{c}$ and this speed is constant of nature. As a conclusion derived from this postulate, it became evident that time runs differently at high speeds of motion of matter (Einstein, 2004) as well as at gravitational extremes (Hawking, 1998; 2001). These results have turned the notion of the universal clock into a new concept of local clocks being a part of space-time. However, the postulate of the invariance of the speed of light does not reveal the fundamental mechanisms that would explain why the speed of light (the group speed) is an invariant constant that cannot be exceeded.

Relativity and quantum mechanics have opened a new page in human understanding of space-time and matter. Both approaches are dealing more accurately with the concept of an observer and its impact on the observation. In his study on the uncertainty principle, Heisenberg wrote "I believe that the existence of the classical 'path' can be pregnantly formulated as follows: The 'path' comes into existence only when we observe it" (Heisenberg, 1927). The essence of this approach implies that the observer changes the observable system by getting into an observation interaction with the observable system.

The Big Bang theory states that the universe and space-time originated with a huge explosion about fourteen (some estimates may vary) billion years ago as an expanding ball of fire that keeps expanding. Some more recent studies extend the concept of the Big Bang to the Self-Reproducing Inflationary Universe (Linde 1994) where "the early universe came through the stage of inflation, exponentially rapid expansion in a kind of unstable vacuum-like state (a state with large energy density, but without elementary particles). Vacuum-like state in inflationary theory usually is associated with a scalar field, which is often called 'the inflation field.' The stage of inflation can be very short, but the universe within this time becomes exponentially large". 
For thousands of years human beings have been learning how to measure time, but we are yet far away from a clear understanding of what make time run. What makes time run slowly in matter and space at near-light speeds and near gravitational extremes and how does time run differently in condensed matter versus in "light" matter and space? Was there a beginning of time? Will there be an end? Though significant progress has been made in modern physics in understanding space-time, the internal structure of time and the fundamental mechanisms that bring time and matter together have yet to be discovered.

To answer these questions first we have to understand what time is. The mystery of time has constantly puzzled researchers and some of them have already made various attempts to understand its internal structure and the mechanisms that make it flow (Barbour, 2001, 1994; Brout, 1987; Green, 2005; Don and William, 1983; Smolin, 1991; Zeh, 1999). Does time exists on its own, is it a part of space, or does time not exist at all (Barbour 2001; 1994; Don and William, 1983; Stenger, 2000).

This study is an attempt to get inside the internal structure of space-time and matter and try to uncover the possible origin and mechanisms that make time run.

\section{Time as a sequence of events:}

The concept of time: It would be a mistake to try to understand time by using the notion of time itself. So, what can we do if every theory in physics that describes motion of matter uses time as a parameter? Let's try to set apart the notion of time and see how the concept of time can be derived from the notion of matter and its transformations. By no means am I trying to remove time from matter and space, rather, just making an attempt to understand its nature and dynamics.

Events and the count of changes: We mention time to refer to the changes in the observable system by mapping the appropriate events in the observable system to some reference events in the reference system. For example, by saying that a tree grew by one meter last year, we refer to the changes in height of the tree as one full circle of the earth around the sun. Likewise, if we say that the distance between two buildings has not changed during the last week, we refer to no changes in the distance between two buildings as seven full rotations of earth around its axis. If we say that a process takes $8.5 \mathrm{sec}$ we refer to all registered events in the process as the reference events in the time registration device, say, one tenth of a second is a reference event that can be registered by the time counting device.
Thus, events in the observable system are referred to events in the reference system (observer). However, the question arises, what kind of events must be referenced. We refer the registered events in the observable system to the measurable events in the reference system. For example, as we observe a process, we register observable events in the processsay, steps of the process we are able to register-and refer them to the time measuring events in the observation system as a count of periods between the events in the observable system-say, clock measuring events-which we consider the accuracy of the clock.

The notion of time: Let's try to analyze time as a measure of the evolution of matter and space. To avoid any possible confusion, let's consider time as a measurable quantity used to specify the order in which events occur in the system. Time periods are being measured by the number of events which precede or follow. Thus, for time to run it needs events to occur Aityan, 2002.

Timeless system: Imagine system $S_{\alpha}$ in which no events are happening. The state of such a system stays unchanged and no time is running inside the system. For time to exist in the system at least one event must take place, otherwise the system has no time at all. The hypothetical system defined above has no grounds for having internal time because it shows no evolution or changes. The system stays "dead" awaiting for events to launch its time mechanism.

Time between the same events from different observation views: Assume an observer from our world (system $\mathrm{S}_{\mathrm{A}}$ )-where time is running in its natural course-has managed to make two observations of another system, $\mathrm{S}_{\mathrm{B}}$, at two different points in time of our world $\left(\mathrm{S}_{\mathrm{A}}\right), \mathrm{t}_{1}$ and $\mathrm{t}_{2}$. Taking into account that no observation can be made without interaction and that interaction generates events, a number of events must be generated by that observation. For the sake of simplicity, assume that every observation generates a single event. Let's call it an "observation event." Thus from the perspective of our world, the time elapsed between these two observations is equal to $t_{2}-t_{1}$; that can be a second, day, month, year or any other period of time from the perspective of system $\mathrm{S}_{\mathrm{A}}$. Assume that only a single event separates these two observations in system $\mathrm{S}_{\mathrm{B}}$, or in other words, just a single bit or quantum of time has elapsed in $S_{B}$ between two observation events in $\mathrm{S}_{\mathrm{A}}$.

Time in different systems: The above example illustrates that time driven by events runs 
fundamentally differently in different systems. This conclusion is completely in synch with the space-time concept contemplated in the theory of relativity. However, there is a conceptual difference between these two approaches. While relativity just ascertains the fact of different time scales in different systems move at different speeds, event-based time describes the internal mechanism that makes time run differently. This mechanism is responsible for time itself and for the differences in the clocks in different systems. Time in each system is being generated by the events in that system, while near-light speeds or super-gravitational forces could be just one of the factors causing variations in the occurrence of events that generate different courses of time in different systems.

For a more general example, let's consider an interaction between two quasi-independent systems, $S_{\alpha}$ and $S_{\beta}$, which engages a much smaller number of events than the number of events occurring within each system in the course of interaction between the systems. If the number of internal events, $T_{\alpha}$ and $T_{\beta}$, between two consecutive interactions between systems $S_{\alpha}$ and $S_{\beta}$ is different, say $T_{\alpha}>T_{\beta}$, then the internal time that elapsed between such interactions in $S_{\alpha}$ is $T_{\alpha}$ which is a longer time than the internal time $T_{\beta}$ that elapsed in $S_{\beta}$ between the same interactions.

Definitions of event and time: Let's assume that there is no time at all. Matter exists and is permanently changing its state. Let's call the change of state an event.

Event $\mathrm{e}_{\mathrm{km}}^{\alpha}$ is a single transition between two states $\mathrm{s}_{\mathrm{k}}^{\alpha}$ and $\mathrm{s}_{\mathrm{m}}^{\alpha}$ of system $\mathrm{S}_{\alpha}$ with no intermediate states between $s_{\mathrm{k}}^{\alpha}$ and $\mathrm{s}_{\mathrm{m}}^{\alpha}$. An illustration of an event is shown in Fig. 1.

Time in system $S_{\alpha}$ is a running count of events that occur in the system. Thus time is just the count of events as shown in Fig. 2. Events can be ordered by only the "next/previous" relationship that constitutes a sequence of events. We will use sign " $\triangleleft$ " to show the "next/previous" relationship. For example, $\mathrm{e}_{\mathrm{k}, \mathrm{k}+1}^{\alpha} \triangleleft \mathrm{e}_{\mathrm{k}+1, k+2}^{\alpha}$ means that event $\mathrm{e}_{\mathrm{k}+1, k+2}^{\alpha}$ is next to $\mathrm{e}_{\mathrm{k}, \mathrm{k}+1}^{\alpha}$ or, vice versa, event $e_{k, k+1}^{\alpha}$ is previous to $e_{k+1, k+2}^{\alpha}$. Let's call such "neighboring" events close events. In other words, there are no other events between close events, at least within the current accuracy of measurement. It may happen that as we increase the precision of event registration, more events will be registered in the system and some previously deemed close events will no longer be close. Thus the notion of close events is subject to the precision of measurement.

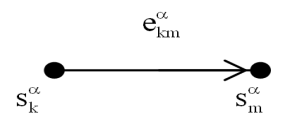

Fig. 1: An event

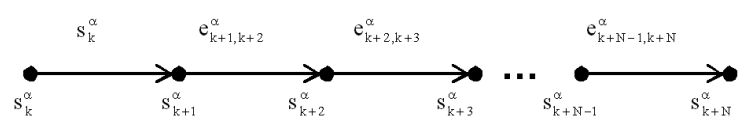

Fig. 2: Sequential events

Any two events in a system can be ordered by " $<$ ". For example, $\mathrm{e}_{\mathrm{k}, \mathrm{k}+1}^{\alpha}<\mathrm{e}_{\mathrm{k}+2, \mathrm{k}+3}^{\alpha}$ means that $\mathrm{e}_{\mathrm{k}, \mathrm{k}+1}^{\alpha}$ preceded event $\mathrm{e}_{\mathrm{k}+2, \mathrm{k}+3}^{\alpha}$, but other events may occur between them.

The distance between any two close events shown in Fig. 2 has no meaning, no value and cannot be measured within the system itself. The fact that some distance, either equal or different, is shown between the events in Fig. 2 is just for illustrative purposes. Every event is a single quantum of time. The time, $\mathrm{t}_{\alpha}\left(\mathrm{e}_{\mathrm{k}, \mathrm{k}+1}^{\alpha}, \mathrm{e}_{\mathrm{k}+\mathrm{N}-1, \mathrm{k}+\mathrm{N}}^{\alpha}\right)$, that elapsed in system $\mathrm{S}_{\alpha}$ between events $\mathrm{e}_{\mathrm{k}, \mathrm{k}+1}^{\alpha}$ and $\mathrm{e}_{\mathrm{k}+\mathrm{N}-1, \mathrm{k}+\mathrm{N}}^{\alpha}$ by definition is a count of the sequential events that occurred between the former mentioned events, i.e. Eq. 1:

$\mathrm{t}_{\alpha}\left(\mathrm{e}_{\mathrm{k}, \mathrm{k+1}}^{\alpha}, \mathrm{e}_{\mathrm{k}+\mathrm{N}-1, \mathrm{k}+\mathrm{N}}^{\alpha}\right)=\mathrm{T}_{\mathrm{e}_{k, k+1}^{\alpha}}^{\mathrm{e}_{k+\mathrm{N}-1, k+\mathrm{N}}^{\alpha}}$

where, $\mathrm{T}_{\mathrm{e}_{k, k+1}^{\alpha}}^{\mathrm{e}_{\kappa+N-1, k+N}^{\alpha}}$ is a count of the sequential events that occurred between events $e_{k, k+1}^{\alpha}$ and $e_{k+N-1, k+N}^{\alpha}$ which is equal to $\mathrm{N}$ in the example shown in Fig. 2.

Observation path: Consider system $S_{\alpha}$ and all events occurring in the system. For the sake of simplicity, we presume that all events are measurable by their energy level and we will use notation $\mathrm{e}_{\mathrm{k}}^{\alpha}$ for $\mathrm{e}_{\mathrm{k}, \mathrm{k}+1}^{\alpha}$. Let's mark the events we want to track or observe in system $S_{\alpha}$ as an observer. We call such events observation events. The observation events marked by crosses in Fig. 3 constitute the observation path:

$$
\Omega_{\alpha}(\mathrm{i}, \mathrm{i}+\mathrm{K})=\left(\mathrm{O}_{\mathrm{i}}^{\alpha}, \mathrm{O}_{\mathrm{i}+1}^{\alpha}, \mathrm{O}_{\mathrm{i}+2}^{\alpha}, \ldots, \mathrm{O}_{\mathrm{i}+\mathrm{K}}^{\alpha}\right)
$$

The observation path measures time between the observations, thus $\mathrm{t}_{\alpha}\left(\mathrm{O}_{\mathrm{i}}^{\alpha}, \mathrm{O}_{\mathrm{i}+1}^{\alpha}\right)$ is the time between observation $\mathrm{O}_{\mathrm{i}}^{\alpha}$ and $\mathrm{O}_{\mathrm{i}+1}^{\alpha}$. In the example given in Fig. 3 , the time in the observation path runs as follows:

$$
\begin{aligned}
& \mathrm{t}_{\alpha}\left(\mathrm{O}_{\mathrm{i}}^{\alpha}, \mathrm{O}_{\mathrm{i}+1}^{\alpha}\right)=3 \\
& \mathrm{t}_{\alpha}\left(\mathrm{O}_{\mathrm{i}+1}^{\alpha}, \mathrm{O}_{\mathrm{i}+2}^{\alpha}\right)=4 \\
& \mathrm{t}_{\alpha}\left(\mathrm{O}_{\mathrm{i}+\mathrm{K}-1}^{\alpha}, \mathrm{O}_{\mathrm{i}+\mathrm{K}}^{\alpha}\right)=1
\end{aligned}
$$


Thus one can count specific time intervals between observation events in system $S_{\alpha}$ and these periods of time are defined by the events in the system.

Time saturation: Every finite and closed system has a finite number of possible states and hence a finite number of possible events. This leads to the conclusion that such a system may exhaust its time counting capability and consequently must reset its internal time.

Timeless systems: Consider an elementary system that has only one possible state, implying that no events may occur in the system. Such a system has no time at all. For instance, a photon may stay in its current state until the photon is absorbed by some other system. Emission of the photon and its absorption are the only events in the photon's life.

The notion of speed: The notion of speed elaborates changes in geometric space per time. Both geometric space and time space (events) have a discrete nature and thus speed represents changes of the position in geometric space per discrete time interval these changes take place:

$\overline{\mathrm{v}}_{\alpha}=\frac{\overline{\Delta \mathrm{x}}_{\alpha}\left(\mathrm{O}_{\mathrm{i}}^{\alpha}, \mathrm{O}_{\mathrm{i}+1}^{\alpha}\right)}{\mathrm{t}_{\alpha}\left(\mathrm{O}_{\mathrm{i}}^{\alpha}, \mathrm{O}_{\mathrm{i}+1}^{\alpha}\right)}$

where, $\overline{\mathrm{v}}_{\alpha}$ is speed, $\overline{\Delta \mathrm{x}}_{\alpha}\left(\mathrm{O}_{\mathrm{i}}^{\alpha}, \mathrm{O}_{\mathrm{i}+1}^{\alpha}\right)$ is a change of the position in geometric space and $\mathrm{t}_{\alpha}\left(\mathrm{O}_{\mathrm{i}}^{\alpha}, \mathrm{O}_{\mathrm{i}+1}^{\alpha}\right)$ is the time interval between observations $\mathrm{O}_{\mathrm{i}}^{\alpha}$ and $\mathrm{O}_{\mathrm{i}+1}^{\alpha}$. Time interval $\mathrm{t}_{\alpha}\left(\mathrm{O}_{\mathrm{i}}^{\alpha}, \mathrm{O}_{\mathrm{i}+1}^{\alpha}\right)$ represents the minimum time between two close observation events because there are no observations between observations $\mathrm{O}_{\mathrm{i}}^{\alpha}$ and $\mathrm{O}_{\mathrm{i}+1}^{\alpha}$. One of the conclusions of the notion of speed given above is that the commonly accepted definition of speed as $\overline{\mathrm{v}}=\frac{\mathrm{d} \overline{\mathrm{x}}}{\mathrm{dt}}=\lim _{\Delta \mathrm{t} \rightarrow 0} \frac{\overline{\Delta \mathrm{x}}}{\Delta \mathrm{t}}$ is no longer valid at $\Delta \mathrm{t}<\mathrm{t}_{\alpha}\left(\mathrm{O}_{\mathrm{i}}^{\alpha}, \mathrm{O}_{\mathrm{i}+1}^{\alpha}\right)$ and must be replaced with the definition given in Eq. 3 .

\section{Local time in subsystems:}

Completely compatible systems: Two systems are completely compatible by time if their combined events can be ordered into a single sequence of events.

According to the definition above, two systems, $S_{\alpha}$ and $S_{\beta}$, are completely compatible by time if the "next/previous" order can be set to all their combined events. In other words, both systems constitute a single system in terms of time. Assume that system $S_{\alpha}$ shown in Fig. 3 is combined with system $S_{\beta}$ shown in Fig. 4 to define a new joint system $\mathrm{S}=\mathrm{S}_{\alpha} \otimes \mathrm{S}_{\beta}$ as shown in Fig. 5 .

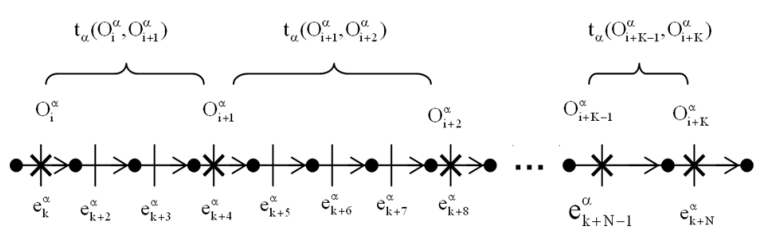

Fig. 3: Observation events

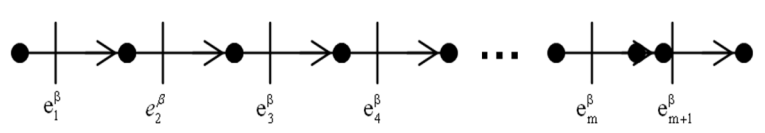

Fig. 4: System $S_{\beta}$

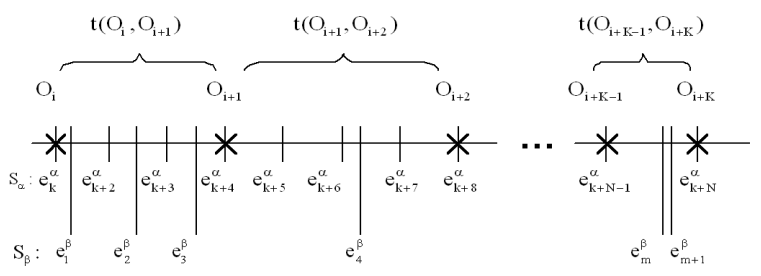

Fig. 5: Completely compatible systems $S_{\alpha}$ and $S_{\beta}$

Completely compatible systems can share their time and the time in a combined system, $\mathrm{S}=\mathrm{S}_{\alpha \beta}=\mathrm{S}_{\alpha} \otimes \mathrm{S}_{\beta}$, is a superposition of times in system $S_{\alpha}$ and system $S_{\beta}$. Thus time measured in the observation path of the combined system $\mathrm{S}$ runs as Eq. 4:

$$
\begin{aligned}
& t_{\alpha \beta}\left(O_{i}^{\alpha \beta}, O_{i+1}^{\alpha \beta}\right)=6 \\
& t_{\alpha \beta}\left(O_{i+1}^{\alpha \beta}, O_{i+2}^{\alpha \beta}\right)=5 \\
& t_{\alpha \beta}\left(O_{i+K-1}^{\alpha \beta}, O_{i+K}^{\alpha \beta}\right)=3
\end{aligned}
$$

In contrast to the time specific to $S_{\alpha}$ only that runs as Eq. 5:

$$
\begin{aligned}
& \mathrm{t}_{\alpha}\left(\mathrm{O}_{\mathrm{i}}^{\alpha}, \mathrm{O}_{\mathrm{i}+1}^{\alpha}\right)=3 \\
& \mathrm{t}_{\alpha}\left(\mathrm{O}_{\mathrm{i}+1}^{\alpha}, \mathrm{O}_{\mathrm{i}+2}^{\alpha}\right)=4 \\
& \mathrm{t}_{\alpha}\left(\mathrm{O}_{\mathrm{i}+\mathrm{K}-1}^{\alpha}, \mathrm{O}_{\mathrm{i}+\mathrm{K}}^{\alpha}\right)=1
\end{aligned}
$$

as shown in Eq. 2. For the time between systems $S_{\alpha}$ and $S_{\beta}$ to be shared, every event in the combined system must be observable from both subsystems.

Parallel (Non-Compatible) systems: Two systems, $\mathrm{S}_{\alpha}$ and $S_{\beta}$, are parallel (non-compatible) by time if none of the events of system $S_{\alpha}$ can be ordered as "next/previous" with any of the events in system $S_{\beta}$ and vice versa.

As follows from the definition of parallel systems, time in parallel systems cannot be tied up to one another due to the incompatibility of their events order. The events in parallel systems cannot be cross-ordered. 
Phy. Intl. 2 (2): 40-49, 2011

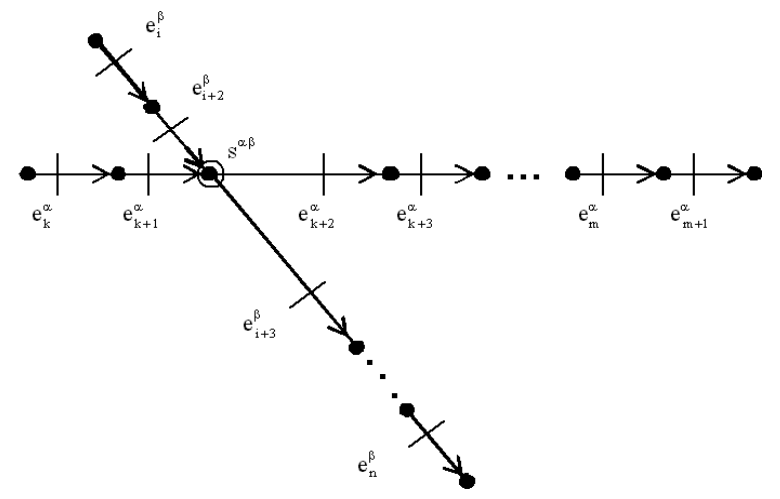

Fig. 6: Orthogonal systems $S_{\alpha}$ and $S_{\beta}$

For example, if no other information in available, systems $S_{\alpha}$ and $S_{\beta}$ shown in Fig. 3 and 4 are parallel. However, as soon as an observer identifies both parallel systems the observer itself becomes a link between the systems through the observation events that makes the initially parallel systems no longer parallel. We will come back to analyze this phenomenon later.

In parallel systems there is no way to say which one of two events in different systems occurred earlier or later than the other one.

Orthogonal (Partially Compatible) systems: Two systems are orthogonal (partially compatible by time) if the systems are not completely compatible by time but at least one event of one of the systems can be ordered as "next/previous" with at least one of the events of the other system. An example of orthogonal systems is shown in Fig. 6. Both systems share a common state, $\mathrm{s}^{\mathrm{a} \beta}$, that allows for a "next/previous" order between event $e_{i+2}^{\beta}$ of system $S_{\beta}$ and event $e_{k+2}^{\alpha}$ of system $S_{\alpha}$ as well as between event $e_{k+1}^{\alpha}$ of system $S_{\alpha}$ and event $e_{i+3}^{\beta}$ of system $S_{\beta}$. Though time in systems $S_{\alpha}$ and $S_{\beta}$ are basically incompatible, the history of both systems can be compared with reference to event $e_{3}^{\beta}$ i.e. can be referred to the common state $s^{a \beta}$ that belongs to both $S_{\alpha}$ and $S_{\beta}$ as a reference point. Such "intersection" creates a common point of reference in the history of the systems incompatible by time. Though it is impossible to say which event, for example $\mathrm{e}_{\mathrm{m}}^{\alpha}$ or $\mathrm{e}_{\mathrm{n}}^{\beta}$, occurred earlier or later than the other, one can easily say that event $e_{n}^{\beta}$ in $S_{\beta}$ follows event $e_{k}^{\alpha}$ in $S_{\alpha}$. Thus in orthogonal systems some events from different systems can be ordered by time while some events cannot.

Time viewed from different systems: Consider two subsystems, $S_{a}$ and $S_{\beta}$, that interact only by two observation events, i.e., they share two common states as shown in Fig. 7.

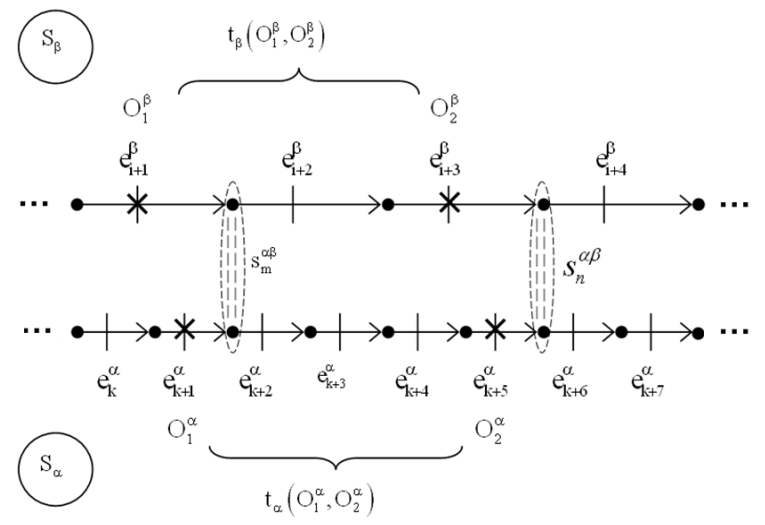

Fig. 7: Time in two systems

Systems $S_{\alpha}$ and $S_{\beta}$ share two common states, $\mathrm{s}_{\mathrm{m}}^{\alpha \beta}$ and $\mathrm{s}_{\mathrm{n}}^{\alpha \beta}$. These common states may occur as a result of interaction between $S_{\alpha}$ and $S_{\beta}$. Suppose observation events in system $\mathrm{S}_{\alpha}$ are $\mathrm{O}_{1}^{\alpha}$, which is $\mathrm{e}_{\mathrm{k}+1}^{\alpha}$ and $\mathrm{O}_{2}^{\alpha}$, which is $\mathrm{e}_{\mathrm{k}+5}^{\alpha}$. Similarly, suppose observation events in system $S_{\beta}$ are $O_{1}^{\beta}$, which is $e_{i+1}^{\beta}$ and $O_{2}^{\beta}$, which is $e_{i+3}^{\beta}$. With no other interactions between the systems they have only two cross-time reference points each: $\mathrm{O}_{1}^{\alpha}$ and $\mathrm{O}_{2}^{\alpha}$ for $\mathrm{S}_{\alpha}$ and $\mathrm{O}_{1}^{\beta}$ and $\mathrm{O}_{2}^{\beta}$ for $\mathrm{S}_{\beta}$. This means that the following cross-order of events can be established:

$\mathrm{e}_{\mathrm{k}+1}^{\alpha} \triangleright \mathrm{e}_{\mathrm{i}+2}^{\beta}$ and $\mathrm{e}_{\mathrm{k}+5}^{\alpha} \triangleright \mathrm{e}_{\mathrm{i}+4}^{\beta}$ for $\mathrm{S}_{\alpha} \rightarrow \mathrm{S}_{\beta}$

and

$\mathrm{e}_{\mathrm{i}}^{\beta} \triangleright \mathrm{e}_{\mathrm{k}+2}^{\alpha}$ and $\mathrm{e}_{\mathrm{i}+3}^{\beta} \triangleright \mathrm{e}_{\mathrm{k}+6}^{\alpha}$ for $\mathrm{S}_{\beta} \rightarrow \mathrm{S}_{\alpha}$

where, " $\rightarrow$ " denotes the direction of view. Thus from the perspective of $S_{\alpha}$, four events $\left\{\mathrm{e}_{\mathrm{k}+2}^{\alpha}, \mathrm{e}_{\mathrm{k}+3}^{\alpha}, \mathrm{e}_{\mathrm{k}+4}^{\alpha}, \mathrm{e}_{\mathrm{k}+5}^{\alpha}\right\}$ occurred between the measurements, while from the perspective of $S_{\beta}$, only two events $\left\{e_{i+2}^{\beta}, e_{i+3}^{\beta}\right\}$ occurred between the same reference points, i.e.:

$$
\begin{aligned}
& \mathrm{t}_{\alpha}\left(\mathrm{O}_{1}^{\alpha}, \mathrm{O}_{2}^{\alpha}\right)=4 \\
& \mathrm{t}_{\beta}\left(\mathrm{O}_{1}^{\beta}, O_{2}^{\beta}\right)=2
\end{aligned}
$$

Eq. 6 explicitly shows that time is running differently in $S_{\alpha}$ and $S_{\beta}$ and different counts of time elapsed between two mutual observations. There are no other time references between these two systems. For example, it is impossible to set any order between events $\mathrm{e}_{\mathrm{k}+3}^{\alpha}$ and $\mathrm{e}_{\mathrm{i}+2}^{\beta}$.

Time absorption: As two systems interact, they add interaction events on top of their internal events and 
hence increase the number of events in each system. Thus every interaction between two systems adds up to the time count in each of them. One can say that each of the interacting systems observes the other if the system is capable of counting time. Consider two independent systems, $S_{\alpha}$ and $S_{\beta}$ and some time intervals in those systems Eq. 7:

$$
\begin{aligned}
& \mathrm{t}_{\alpha}\left(\mathrm{e}_{\mathrm{k} 1}^{\alpha}, \mathrm{e}_{\mathrm{k} 2}^{\alpha}\right)=\mathrm{T}_{\mathrm{e}_{\mathrm{k} 1}^{\alpha}}^{\mathrm{e}_{2}^{\alpha}} \mathrm{t}_{\beta}\left(\mathrm{e}_{\mathrm{m} 1}^{\beta}, \mathrm{e}_{\mathrm{m} 2}^{\beta}\right)=\mathrm{T}_{\mathrm{e}_{\mathrm{m} 1}^{\beta}}^{\mathrm{e}_{\mathrm{m} 2}^{\beta}}
\end{aligned}
$$

Assume that interaction between systems $S_{\alpha}$ and $S_{\beta}$ has generated additional events $\left\{\mathrm{e}_{\mathrm{n} 1}^{\alpha \beta}, \ldots, \mathrm{e}_{\mathrm{n} 2}^{\alpha \beta}\right\}$ between events $\mathrm{e}_{\mathrm{k} 1}^{\alpha}$ and $\mathrm{e}_{\mathrm{k} 2}^{\alpha}$ in $\mathrm{S}_{\alpha}$ and additional events $\left\{\mathrm{e}_{\mathrm{r} 1}^{\beta \alpha}, \ldots, \mathrm{e}_{\mathrm{r} 2}^{\beta \alpha}\right\}$ between events $\mathrm{e}_{\mathrm{m} 1}^{\beta}$ and $\mathrm{e}_{\mathrm{m} 2}^{\beta}$ in $\mathrm{S}_{\beta}$. As a result, the time count in both systems has changed to Eq. 8:

$$
\begin{aligned}
& \tilde{t}_{\alpha}\left(e_{k 1}^{\alpha}, e_{k 2}^{\alpha}\right)=t_{\alpha}\left(e_{k 1}^{\alpha}, e_{k 2}^{\alpha}\right)+t_{\alpha}\left(e_{n 1}^{\alpha \beta}, e_{n 2}^{\alpha \beta}\right)=T_{e_{k 1}^{\alpha 2}}^{e_{k}^{\alpha}}+T_{e_{n 1}^{\alpha \beta}}^{e_{\alpha \beta}^{\alpha \beta}} \\
& \tilde{t}_{\beta}\left(e_{m 1}^{\beta}, e_{m 2}^{\beta}\right)=t_{\beta}\left(e_{m 1}^{\beta}, e_{m 2}^{\beta}\right)+t_{\beta}\left(e_{r 1}^{\beta \alpha}, e_{r 2}^{\beta \alpha}\right)=T_{e_{m 1}^{\beta}}^{e_{m 2}^{\beta}}+T_{e_{r 1}^{\beta}}^{e_{2}^{\beta \alpha}}
\end{aligned}
$$

Let's call this phenomenon time absorption. It is clear that with more interaction between these systems, more time is being added to both of them.

\section{Observation and observer:}

Observation, observer and existence: Anything we know about our world must be observed otherwise we have no chance to know about it. For example, if an entire other world exists somewhere in the universeeven close to us-but we are unable to interact with it, then it does not exist for us until we find a way to interact with that other world and observe it. By observation we mean more than just explicit observation, i.e., an observation made directly by our senses. Some observations are implicit; for example, humans do not explicitly observe the chemical content of food they eat but the chemical content impacts the human metabolism and finally the human body. In this study, observation refers to a general meaning of interaction without differentiating explicit and implicit observation.

We understand the observer as a system that is able to interact with the observable system and does so by explicit or implicit observation.

Though the major focus of this study is on the observation of time, the concept of observation and observer can be applied to any other types of observation. This concept is completely in line with the concept of observation and observer commonly accepted in modern physics.

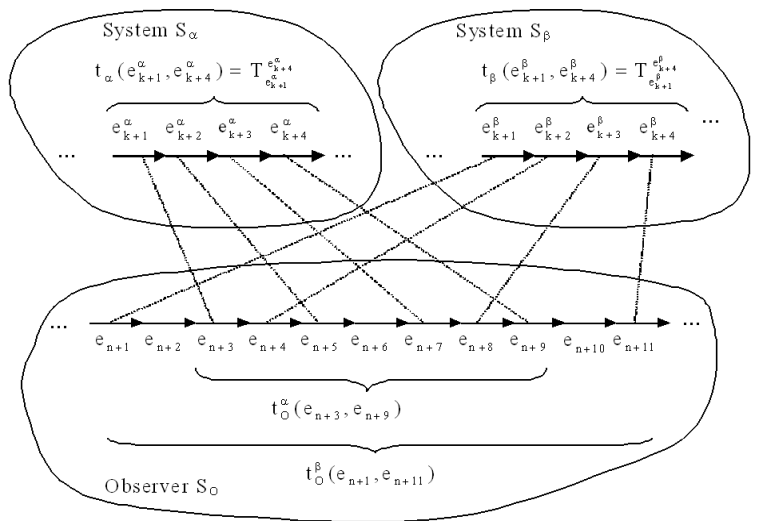

Fig. 8: Observation of two systems

Observing time in two systems: Imagine two absolutely identical but separate and not interacting systems, $S_{\alpha}$ and $S_{\beta}$, that go through the sequence of events as shown in Fig. 8.

Assume that the sequence of events in system $\mathrm{S}_{\alpha}$ : $\left\{\mathrm{e}_{\mathrm{k}+1}^{\alpha}, \mathrm{e}_{\mathrm{k}+2}^{\alpha}, \mathrm{e}_{\mathrm{k}+3}^{\alpha}, \mathrm{e}_{\mathrm{k}+4}^{\alpha}\right\}$ is identical to the sequence of events in system $\mathrm{S}_{\beta}:\left\{\mathrm{e}_{\mathrm{k}+1}^{\beta}, \mathrm{e}_{\mathrm{k}+2}^{\beta}, \mathrm{e}_{\mathrm{k}+3}^{\beta}, \mathrm{e}_{\mathrm{k}+4}^{\beta}\right\}$. It implies that the internal time in system $S_{\alpha}$ from the view of system $S_{\alpha}$ runs identically to the internal time in system $S_{\beta}$ from the view of system $S_{\beta}$ Eq. 9:

$\mathrm{t}_{\alpha}\left(\mathrm{e}_{\mathrm{k}+1}^{\alpha}, \mathrm{e}_{\mathrm{k}+2}^{\alpha}, \mathrm{e}_{\mathrm{k}+3}^{\alpha}, \mathrm{e}_{\mathrm{k}+4}^{\alpha}\right)=\mathrm{t}_{\beta}\left(\mathrm{e}_{\mathrm{k}+1}^{\beta}, \mathrm{e}_{\mathrm{k}+2}^{\beta}, \mathrm{e}_{\mathrm{k}+3}^{\beta}, \mathrm{e}_{\mathrm{k}+4}^{\beta}\right)=4$

For the sake of simplicity, let's assume that every event in $S_{\alpha}$ and $S_{\beta}$ results in the emission of a particle, say, a photon that can be observed by system $S_{0}$. Each time for $S_{\alpha}$ and $S_{\beta}$ from system $S_{0}$ runs differently for $\mathrm{S}_{\alpha}$ and $\mathrm{S}_{\beta}$ :

$\mathrm{t}_{\mathrm{O}}^{\alpha}\left(\mathrm{e}_{\mathrm{k}+1}^{\alpha}, \mathrm{e}_{\mathrm{k}+2}^{\alpha}, \mathrm{e}_{\mathrm{k}+3}^{\alpha}, \mathrm{e}_{\mathrm{k}+4}^{\alpha}\right)=\mathrm{t}_{\mathrm{o}}\left(\mathrm{e}_{\mathrm{n}+3}, \ldots, \mathrm{e}_{\mathrm{n}+9}\right)=7$
$\mathrm{t}_{\mathrm{O}}^{\beta}\left(\mathrm{e}_{\mathrm{k}+1}^{\beta}, \mathrm{e}_{\mathrm{k}+2}^{\beta}, \mathrm{e}_{\mathrm{k}+3}^{\beta}, \mathrm{e}_{\mathrm{k}+4}^{\beta}\right)=\mathrm{t}_{\mathrm{O}}\left(\mathrm{e}_{\mathrm{n}+1}, \ldots, \mathrm{e}_{\mathrm{n}+11}\right)=11$

Furthermore, every event in $S_{\alpha}$ is registered with every other event in observer $S_{0}$, i.e., the observation shows the same time periods between the events observed in $S_{\alpha}$ while the time periods observed between events in $S_{\beta}$ are different in $S_{0}$. The time periods between the events in $S_{\alpha}$ observed by $S_{0}$ run as $(2,2,2)$ while the time between the events in $S_{\beta}$ observed by $S_{0}$ run as $(3,4,3)$.

Thus two identical systems, $S_{\alpha}$ and $S_{\beta}$, that have identical local internal times may be observed with different times by the observer. The scale of processes occurring in two identical systems may also be skewed by the fact of observation. 
Impact of observations on the local time in systems: Every observation adds up events in both the observer system and the observable system. In the macro world, the number of observation events is negligibly smaller than the number of bulk (internal) events in every system. Therefore, in the macro world we never register any impact of the observation on time. However, if the number of bulk events in the observer system is comparable or greater than the number of observation events generated by the observation, then the impact of the observation must start playing a significant role in the flow of time.

Consider two systems, $S_{\alpha}$ and $S_{\beta}$, where $S_{\beta}$ is an observer and $S_{\alpha}$ is an observable system. Fundamentally, there is no difference between an observer and an observable system because both systems are observing each other as they interact. In the present example we have identified the observer and the observable system just for convenience, so all further considerations are also applicable in the opposite direction.

Weak impact observations: Assume that the number of internal bulk events in observer $S_{\beta}$ is much higher than the number of observation events in the observer. In this case, Eq. 11 shows the differences in time, similar to Eq. 10:

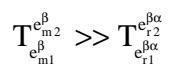

that leads to Eq. 12:

$$
\tilde{\mathrm{t}}_{\beta}\left(\mathrm{e}_{\mathrm{m} 1}^{\beta}, \mathrm{e}_{\mathrm{m} 2}^{\beta}\right) \approx \mathrm{t}_{\beta}\left(\mathrm{e}_{\mathrm{m} 1}^{\beta}, \mathrm{e}_{\mathrm{m} 2}^{\beta}\right)
$$

This implies virtually no changes in the time course of the observer generated by the observation.

Equal impact observations: Assume that the number of internal bulk events in observer $S_{\beta}$ is comparable with the number of observation events in the observer. In this case, as with Eq.10 and 13:

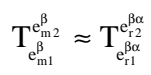

that leads to Eq. 14-16:

$$
\tilde{t}_{\beta}\left(e_{m 1}^{\beta}, e_{m 2}^{\beta}\right)=t_{\beta}\left(e_{m 1}^{\beta}, e_{m 2}^{\beta}\right)+t_{\beta}\left(e_{r 1}^{\beta \alpha}, e_{r 2}^{\beta \alpha}\right) \approx 2 t_{\beta}\left(e_{m 1}^{\beta}, e_{m 2}^{\beta}\right)
$$

This implies that the time duration in the observer is about to double due to the observation.

Strong impact observations: Assume that the number of internal bulk events in observer $S_{\beta}$ is much less than the number of observation events in the observer. In this case, as with Eq.10:

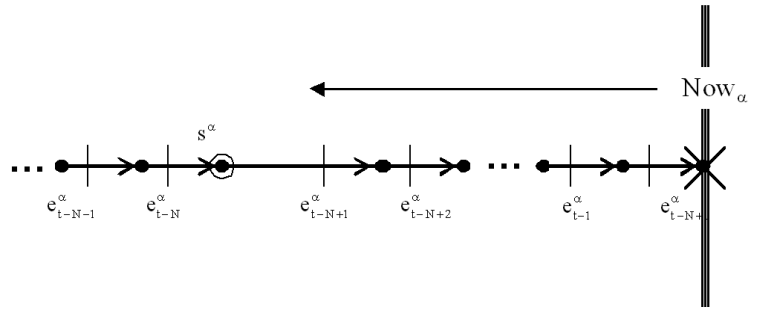

Fig. 9: Back tracking along the time thread by view from Now

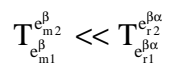

Leads to:

$\tilde{\mathrm{t}}_{\beta}\left(\mathrm{e}_{\mathrm{m} 1}^{\beta}, \mathrm{e}_{\mathrm{m} 2}^{\beta}\right) \approx \mathrm{t}_{\beta}\left(\mathrm{e}_{\mathrm{r} 1}^{\beta \alpha}, \mathrm{e}_{\mathrm{r} 2}^{\beta \alpha}\right)>\mathrm{t}_{\beta}\left(\mathrm{e}_{\mathrm{m} 1}^{\beta}, \mathrm{e}_{\mathrm{m} 2}^{\beta}\right)$

This implies that the time course in observer $S_{\beta}$ was dramatically changed due to the observation.

Normal observations: The fact that in our normal dayto-day observations we do not register the impact of each observation on time just implies that the number of bulk events in our world is much higher than the observation events we are registering. As we start coming to extreme areas where the number of observation events becomes comparable with or higher than the number of bulk events, we most likely start registering an impact of the observations on the system clock.

\section{Multidimensionality of time:}

Virtual time threads: At any particular moment (event $\mathrm{e}_{\mathrm{t}}^{\alpha}$ ) in system $\mathrm{S}_{\alpha}$, events can be tracked back to past events along the time thread that led to the current state of the system as shown in Fig. 9. In other words, the system itself knows its history. However, an observer is capable of knowing the history of system $S_{\alpha}$ only to the degree of observation events that link $S_{\alpha}$ with the observer. If an observer places himself at one of the past states of system $S_{\alpha}$ and tries to predict the next event, he will face multiple possibilities due to the uncertainty or probabilistic nature of the events in the system as shown in Fig. 10.

Thus from the perspective of $\mathrm{Now}_{\mathrm{a}}$, event $\mathrm{e}_{\mathrm{t}-\mathrm{N}}^{\alpha}$ is a certain event that was a member of the time thread leading to $\mathrm{Now}_{\alpha}$. On the other hand, from the perspective of event $e_{t-\mathrm{N}}^{\alpha}$, several different threads $\langle\alpha, \beta, \gamma, \ldots\rangle$ have chances to be realized and thread $\langle\alpha\rangle$ leading to $\mathrm{Now}_{\alpha}$ is just one of them. For the observer located at Now $_{a}$, all other threads $\langle\beta, \gamma, \ldots\rangle$ except thread $\langle\alpha\rangle$ shown in Fig. 10 can be considered virtual threads, i.e., the threads that were possible but not realized from the perspective of $\mathrm{Now}_{\alpha}$. 


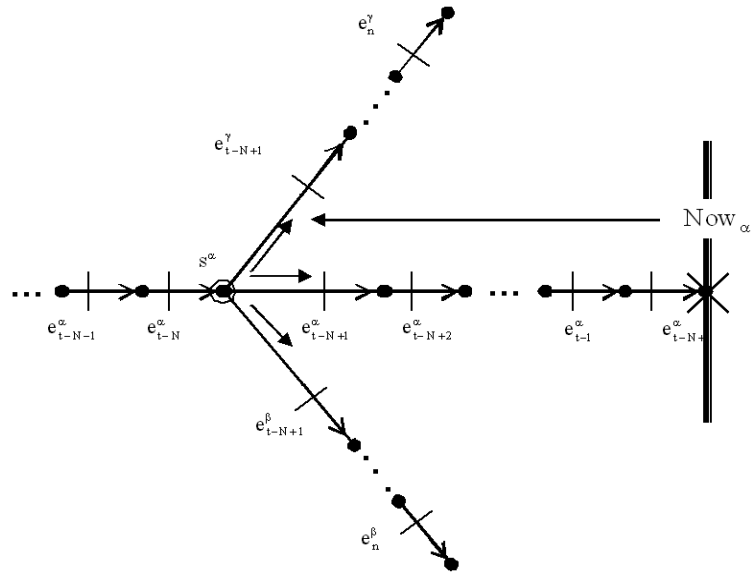

Fig. 10: Dual view at the causality thread

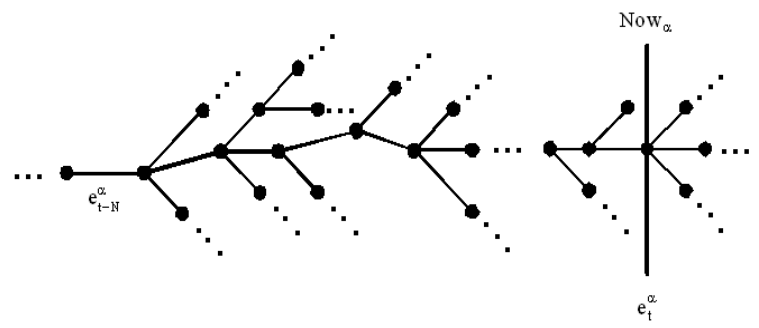

Fig. 11: Multiplicity of virtual threads

All these threads define orthogonal systems because they are not completely compatible by time but have at least a common reference at $\mathrm{e}_{\mathrm{t}-\mathrm{N}}^{\alpha}$.

In general, the virtual threads originated from $\mathrm{e}_{\mathrm{t}-\mathrm{N}}^{\alpha}$ are shown in Fig. 11 which illustrates multiplicity of virtual threads. Virtual thread $\langle\alpha\rangle$ is shown in Fig. 11 in bold lines. Let's call the virtual threads virtual worlds.

To summarize the different perspectives from different positions of the observer on thread $\langle\alpha\rangle$, one must note that from the perspective of $\operatorname{Now}_{\alpha}$ event $\mathrm{e}_{\mathrm{t}-\mathrm{N}}^{\alpha}$ is a definite ancestor of $\operatorname{Now}_{\alpha}$ while any $\operatorname{Now}_{X<X=\alpha, \beta, \gamma \ldots>}$ is a virtual descendant of event $e_{t-\mathrm{N}}^{\alpha}$ from the perspective of event $e_{t-\mathrm{N}}^{\alpha}$.

Dimensions of time: Every virtual time thread represents a new dimension of time. The time in a virtual time thread is not compatible with the time in any other virtual time thread except for the common point of reference or points of reference represented by the common event or events in the past from which these threads were originated. It implies that all virtual time threads that originated from any event have this common "time" point of reference, while future events in the time threads cannot be compared or ordered with future events in the other threads unless these threads will intersect in the future.

Assuming that our universe goes through an infinitely large number of events that originate an infinite number of virtual time threads, one can conclude that time has an infinite number of dimensions.

Thus every state of matter in our universe opens a possibility for multiple virtual events that create virtual time threads. Then, every virtual time thread may split into multiple time threads at every further event. In a finite system the number of virtual threads is large but finite, while in an infinite system the power of the set of all virtual threads is infinite $\left\{2^{\mathrm{n}}\right\}$ which makes it uncountable or continuum.

As long as disorder increases as the universe evolves, it creates more and more virtual threads and hence dimensions of time.

The past and the future: As mentioned above in a discussion on virtual time threads, all past events (or previous events) on the time thread viewed back from a position on a time thread, such as $\mathrm{Now}_{\alpha}$, have occurred with certainty while future events are yet uncertain. This makes a substantial difference between the past and the future. One can say that the past is a sequence of events that have been realized while the future is a possibility of events that may occur. Thus any current observation point is a break point between the past and the future, which have quite a different nature.

I would like to avoid creating a wrong impression that the future cannot be predicted by the application of this theory. Any observable process is a sequence of observation events as shown in Fig. 3. These observation events are a small subset of the other events, referred to as bulk events, happening in the universe. Thus in predicting a future under the assumption that the chosen observation events have a negligible impact on the bulk events in a system, we can consider the bulk events to be the main time generating events that set up a reference "clock" for the observation events in the process as discussed above in this study. It is clear that such clocks are basically measuring different counts of time depending on the process, environment, points of reference and specific conditions of matter. If a process significantly impacts the bulk events in a system by significantly changing the number of bulk events, then setting up a reference "clock" in the system may become a more complicated task.

The implication is that such local clocks can be different for different systems, subsystems and observations and that there is no universal clock for many reasons, at the very least because there is no universal observer. 
The arrow of time: As noted by Stephen Hawking, "Disorder increases with time because we measure time in the direction in which disorder increases" (Hawking, 1998).

The event-based approach defines arrow of time as the "next/previous" relationship between the events in each virtual time thread. However, there is no such notion as an arrow of time between different time threads for the events that cannot be ordered into the "next/previous" relationship. It is quite possible that time may not be obeying a straight arrow of time between orthogonal and partially orthogonal time threads.

For the finite and closed systems, the arrow of time may reset as time in the system saturates and that brings the system to some previous and pre-existed state. Such an effect may change or reverse the arrow of time within the same virtual time thread. With an interaction between different time threads, such effects may occur even without saturation of time in any specific time thread.

Transition to the continuous time: To extend the definition of time given in this study to the world where we belong, one can say that most measurements we make represent the observation paths upon a sequence of an enormous number of other events occurring in the system that set up the "clock" for the system. Let's call these other events "bulk events". These bulk events make us believe in the existence of the "universal clock" ticking with no regard to the processes we measure. However, as soon as we start pushing the envelope to the extreme, a more accurate understanding and interpretation of the notion of time must replace such a traditional understanding of time.

Though time is measured as a count of events and has an inherently discrete nature, actually, time can be treated as a continuous parameter in large systems due to an enormously large number of diverse bulk events per every measurement. The transition from a discrete to a continuous description is typical for physics and other sciences, for instance, the transition from quantum mechanics to classical mechanics or the application of the continuous approach to population dynamics even though the population consists of a discrete number of individuals. Thus continuous time is just a convenient approximation for the fundamentally discrete (quantum) time. In this regard, we have to be ready to find out that even continuous time runs differently in different systems under much broader conditions than has been defined by relativity effects.

\section{CONCLUSION}

This study has addressed the nature and structure of time as an immanent count of the evolution of matter and space. The main focus was on the foundations of the event-based theory of time that explains how time originates and runs. Though all of us have already been accustomed to the discrete (quantum) nature of matter and space in modern physics, we still keep considering time to be a continuous and scalar parameter. The approach taken in this study makes time a multidimensional component of the multidimensional space and matter. The greatest mystery of the notion of time is that there is no time at all. It is a derivative concept of the motion of matter. Matter creates time in its evolution as a measure of changes in matter and space.

This concept of time is believed to provide a new angle of viewing space-time as a unified entity. It is expected to find experimental proofs of the theory in astronomy and cosmology, in elementary particles and at low temperatures where the number of bulk events starts deviating from what we have in our normal world. For example, a reflection in a cold mirror at a low temperature is expected to show some red shift unlike the same reflection at higher temperatures.

Time originated in the universe at the Big Bang, but it runs differently in different systems in the universe and even differently at different stages of the evolution of the universe. It is quite possible that time at the early stages of the universe ran differently from time in our days and what from today's view would be a split fraction of a second just after the Big Bang would be billions of years from a point of view closer to the time of the Big Bang.

The event-based theory of time presented in this study is believed to be just the first step in a comprehensive study in this area. There are still many questions to be answered and more detailed theory to be developed in line with the concepts of this study. It would be interesting to learn more about the interaction of matter, space and time and its dependence on energy. As long as disorder increases as the universe evolves, the concept of entropy of time should be considered with regard to the arrow of time and multidimensionality of time. It is very important to show how event-based time describes the fundamental laws of physics. It is quite possible that the postulate of invariance of speed of light in a vacuum in the relativity and uncertainty principle in quantum mechanics are based on the quantum (event-based) nature of time. It would not be surprising if universal constants in physics become no longer constants but a reflection of the current state of matter and space. All these and many other questions will be addressed in our future research.

The greatest mystery of the notion of time is that there is no time at all!

\section{ACKNOWLEDGMENT}

I greatly appreciate extremely helpful and challenging discussions with and comments by Professor Octavian Ksenzhek (Ukrainian 
Technological University) whose enthusiasm, support and constructive criticism is impossible to overestimate. I would like to thank Professor Konstantin Vodopyanov (Stanford University, USA) and Professor Alexei Kornyshev (Imperial College, UK) for many stimulating discussions that have pumped a lot of enthusiasm and energy into my work on this topic. I would like to thank Nicole Marsh for her valuable help in the preparation of this paper.

\section{REFERENCES}

Aityan, S.K., 2002. Concept of event driven time, presentation at the Russian Academy of Science.

Barbour, J.B., 2001. The End of Time. The Next Revolution in Physics. 1st Edn., Oxford University Press, Oxford, ISBN-10: 0195145925, pp: 384.

Barbour, J.B., 1994. The timelessness of quantum gravity: 1 . The evidence from the classical theory. Class. Q. Grav. DOI:10.1088/0264-9381/11/12/005

Brout, R., 1987. On the concept of time and the origin of the cosmological temperature. Found. Phys., 17: 603-619. DOI: 10.1007/BF01882790

Einstein, A., 2004. Relativity: The Special and the General Theory. 15th Edn., Barnes and Noble Publishing, New York, ISBN-10: 0760759219, pp: 201.

Greene, B., 2005. The Fabric of the Cosmos: Space, Time and the Texture of Reality. 1st Edn., Penguin Books Ltd, London, ISBN-10: 0141959959, pp: 592.
Hawking, S.W., 2001. The Universe in a Nutshell. 1st Edn., Bantam Books, New York, ISBN: 055380202X pp: 216.

Hawking, S.W., 2009. A Brief History Of Time: From Big Bang to Black Holes. 1st Edn., Transworld, USA., ISBN-10: 1409092364 pp: 256.

Heisenberg, W., 1927. Zeitschrift für Physik. 43: 72-198.

Linde, A., 1994. The Self-Reproducing Inflationary Universe. Sci. Am.

Newton, 1687. Isaac: Principia.

Newton, I. and A.E.S. Reviewer, 1999. The Principia: Mathematical principles of natural philosophy. Physicstoday, 53:73-73. DOI: 10.1063/1.883005

Don, P.N. and W.K. William, 1983. Evolution without evolution: Dynamics described by stationary observables. Phys. Rev., 27: 2885-2892.

Smolin, L., 1991. Space and Time in the Quantum Universe. In: Conceptual Problems of Quantum Gravity, Ashtekar, A. and J.J. Stachel, (Eds.). Springer, Boston, pp: 228-269.

Stenger, V.J., 2000. Timeless Reality: Symmetry, Simplicity and Multiple Universes. 1st Edn., Prometheus Books, Amherst, N.Y., ISBN-10: 1573928593, pp: 396.

Zeh, H.D., 1999. The Physical Basis of the Direction of Time. 3rd Edn., Springer, Berlin, ISBN-10: 3540648658, pp: 228. 